\title{
Access to and use gaps of insecticide- treated nets among communities in Jimma Zone, southwestern Ethiopia: baseline results from malaria education interventions
}

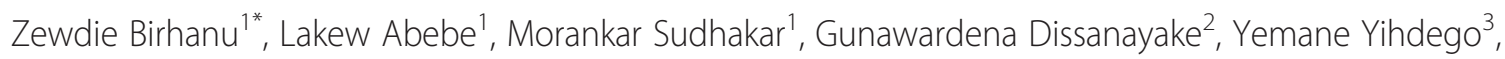
Guda Alemayehu ${ }^{2}$ and Delenasaw Yewhalaw ${ }^{4}$

\begin{abstract}
Background: Malaria remains one of the major public health concerns in Ethiopia. Use of long- lasting insecticidal nets (LLINs) is the country's key malaria prevention and control strategy. This study intended to determine access to and usage gap of LLINs in malaria endemic settings in Southwestern Ethiopia.

Methods: Data were collected from 798 households in three districts (Mana, Kersa and Goma) of Jimma Zone, Southwestern Ethiopia, from December 2013 to January 2014. The data were analyzed using SPSS software package version 17.0. LLINs ownership, access and utilization gap were determined following the procedure developed by Survey and Indicator Task Force of the Roll Back Malaria Monitoring and Evaluation Reference Group. To complement the quantitative data, focus group discussions and interviews were conducted with community groups and key informants.

Results: In this study, $70.9 \%$ (95 \% Cl: 67.8-74.1\%) of the surveyed households had at least one LLIN, and $63.0 \%$ (95\% Cl: 59.6-66.3 \%) had sufficient LLINs for every member of the household. With respect to access, $51.9 \%$ (95\% Cl: 50.5-53.5 \%) of the population had access to LLIN. Only, $38.4 \%$ (95\% Cl: 36.9-39.9\%) had slept under LLIN the previous night with females and children having priority to sleep under LLIN. This gave an overall use to access ratio of $70.2 \%$ which resulted in behavior-driven failure of $29.8 \%$. Of the households with sufficient LLIN access, females (AOR $=1.52 ; 95 \% \mathrm{Cl}: 1.25-1.83 ; P=0.001)$ and children aged $0-4$ years (AOR $=2.28 ; 95 \% \mathrm{Cl}: 1.47-3.53$; $P=0.001$ ) were more likely to use LLINs than other household members. Shape of nets, sleeping arrangements, low risk perception, saving nets for future use, awareness and negligence, and perception of low efficacy of the LLINs contributed to behavioral failures.
\end{abstract}

Conclusions: LLIN use was hampered by lack of ownership and most importantly by behavioral driven gaps. This calls for designing and implementing appropriate behavioral change communication strategies to address behavioral failure. Improving access to LLINs also needs attention. Further, it requires moving beyond the traditional messaging approach for evidence based intervention to address specific needs and gaps.

Keywords: Long lasting insecticide treated net, LLIN ownership, LLIN use, LLIN access, Behavioral change communication, Malaria, Ethiopia

\footnotetext{
* Correspondence: zbkoricha@yahoo.com

'Department of Health Education and Behavioral Sciences, Jimma University,

Jimma, Ethiopia

Full list of author information is available at the end of the article
} 


\section{Background}

Over the last decades, the world has documented a remarkable success in the fight against malaria, especially in sub-Saharan Africa including Ethiopia [1, 2]. Nevertheless, malaria remains a global public health and socio-economic burden. In 2014, the World Health Organization (WHO) estimated that malaria caused 198 million illnesses worldwide leading to approximately 584 000 deaths. Africa is the most affected continent with $90 \%$ of all malaria deaths mainly occur in children less than 5 years of age. In 2013, an estimated 437,000 African children died before their fifth birthday due to malaria [1]. Despite tremendous efforts and commitments, malaria continues to be one of the major health and socioeconomic burdens in Ethiopia [2, 3]. In 2013, there were more than three million confirmed malaria consultations, recorded as the first cause of morbidity $(11.7 \%)$ and the third leading cause of health facility admission (7.8 \%). Likewise, it was the fourth leading cause of health facility consultation (9.6\%) and the second leading cause of health facility admission among under five children. In the same year, malaria accounted for $5.8 \%$ (both confirmed and clinical) death among under five children [3].

Ethiopia has given considerable attention to malaria prevention and control [4]. With sustained universal coverage of key malaria interventions, the country has envisioned eliminating malaria by 2020 [5]. As part of this strategic vision, the government has aimed to achieve malaria elimination in areas with historically low malaria transmission and achieve near zero malaria deaths in all other parts of the country by 2015 [5]. To this effect, the national malaria control program has planned to scale up the coverage and distribution of long-lasting insecticidal nets (LLINs) to $100 \%$ and increase ownership (at least two LLINs per household) in malaria endemic areas, and reach 86 \% LLIN use among pregnant women and under five children by 2015 [4, 5]. Given that this plan is in due date, the government and key malaria partners have updated their strategic plan to attain the long-term goal of worldwide malaria elimination and eventual eradication by 2040-2050. For instance, the President's Malaria Initiative (PMI) Strategy (2015-2020) reaffirms continuing to sustain universal access and use of LLINs to assist PMI supported countries to progress towards elimination and eventual eradication [2].

To ensure universal access, WHO recommends that one LLIN should be distributed for every two people at risk of malaria, and thus, improving access to LLINs should be the first priority [6]. Evidence has shown that there is a high correlation between access and use of LLINs. However, in areas where LLIN use is lower, WHO recommends the roll-out of well-designed behavior-change communication (BCC) interventions [7]. For several years, two main indicators were being used to assess LLIN use; the "proportion of households owning at least one LLIN" and the "proportion of children under five and pregnant women sleeping under LLIN the previous night" [8]. However, these indicators have their own limitations since they consistently show a substantial gap between ownership and actual use of nets by vulnerable groups i.e. children and pregnant women or other family members. Although lack of access to LLIN contributes to non-use, behavior-driven failure also plays a key role [9].

In order to measure access to LLIN in a more appropriate way, the Roll Back Malaria (RBM) recommended two additional core LLIN indicators [10]: the "proportion of households with at least one LLIN for every two people" and the "proportion of the population that has access to an LLIN within the household" [11]. The first indicator is used in combination with the previous indicator "proportion of households with at least one LLIN" to estimate the ownership gap (i.e. households with no or insufficient LLIN) in a better way. The second indicator assists to measure the use gap which is due to behavioral failure. However, access to and use gaps of LLINs have been studied little in Ethiopia. Therefore, this study investigated LLIN access and use gaps among households in three districts of Jimma Zone, Southwestern Ethiopia.

\section{Methods \\ Study setting}

The data were obtained from a larger community based cross-sectional survey conducted during December 2013 to January 2014 in three districts (Mana, Goma and Kersa) of Oromia region. The three districts are parts of Jimma Zone in Oromia. The Zone is located on the geographic coordinates of $7^{\circ} 40^{\prime} 0^{\prime \prime} \mathrm{N}$ latitude and $36^{\circ} 50^{\prime} 0^{\prime \prime}$ E longitude in Oromia, Southwestern Ethiopia. Figure 1 shows map of the study area. The data were collected to establish baseline indicators for malaria education interventions implemented through school students and religious leaders. The three districts were purposively selected based on the burden of malaria and absence of ongoing malaria interventions by Non-Governmental Organizations. Goma district is situated at an altitude that ranges from 1380 to $1680 \mathrm{~m}$, and about $20.1 \%$ of the landmass of the district is swampy. The total population of this district was 213,023 and only $5.9 \%$ of its population were urban dwellers. Mana is a district neighboring to Goma and has a total population of 146,675 where females accounts for $49.1 \%$ and only $3.0 \%$ of the population were residing in urban areas. The altitude of the district ranges from 1470 to $2610 \mathrm{~m}$ above sea level. Likewise, Kersa district is located at altitude that ranges from 1740 to $2660 \mathrm{~m}$. The population of the district was about 165,391 , of whom $49.5 \%$ were females, and $3.28 \%$ of the 


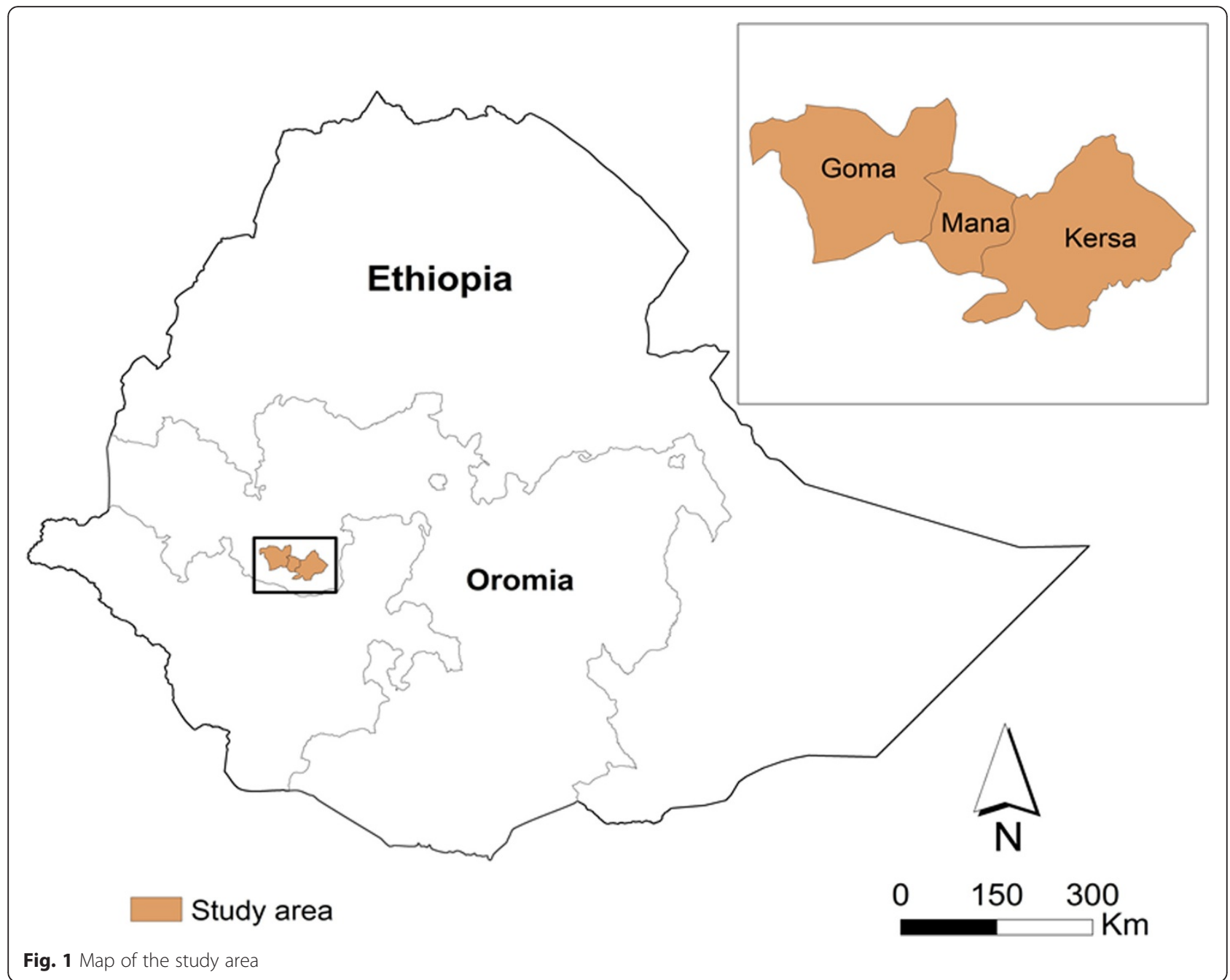

population were living in urban areas. Oromo is the dominant ethnic group and the majority of inhabitants were Muslims in the three districts [12]. The three districts are found in similar geographical and ecological conditions. And, malaria is an endemic in the locality. In Ethiopia, which is also true to the study area, malaria transmission is mostly unstable and seasonal. Peak transmission generally occurs between September and December after the main rainy season (i.e. from June to August). However, in southwestern of Ethiopia where the study area is located, rainfall season often begins earlier in April and the area lacks clearly defined rainfall season. In addition, following a short rainy season in February and March, some minor malaria transmission also occurs in April, May and June. The country also experiences some inter-annual variation in rainfall and temperature leading to a persistent risk of malaria transmission patterns $[5,13,14]$. Comprehensive data are lacking on the pattern of malaria in the study area but one community based study in the adjust district reported a prevalence of $10.5 \%$ where Plasmodium falciparum accounted for $40.9 \%$ [15], and another retrospective study in one of the study district reported that Plasmodium falciparum accounts for $62.4 \%$ of malaria cases [16].

\section{Study design and population}

This baseline study employed a community crosssectional design triangulated with qualitative methods to assess households' behavioral practices on malaria prevention and control in the three districts. However, this article reported LLIN access and use gaps in the study community. The sample size was determined using single population proportion formula $\left(n=Z_{1-\alpha / 2}^{2} \mathrm{p}(1-\mathrm{p}) / \mathrm{d}^{2}\right)$ assuming the proportion of under five children who slept under LLIN during the previous night (55.4\%) [17], $5 \%$ marginal error, $95 \%$ confidence interval, design effect of 2 and $10 \%$ non-response rate. This gave a sample size of 834 households. The households were selected randomly from 13 Gandas (the smallest administrative unit in Oromia, Ethiopia). The number of Gandas was decided 
considering logistic feasibility and resource available for the study. In each district, Gandas with high malaria risk were randomly selected. The stratification of malaria transmission as high risk, in the study districts, generally followed the national guideline which considered elevations below $2000 \mathrm{~m}$ above sea level as high risk area [2, 5]. In addition, some local factors such as malaria case load and availability of water collections for vector breeding were considered to define Gandas as having risk of malaria transmission. Consequently, the number of Gandas with risk of malaria transmission were 20, 16 and 26 in Kersa, Mana and Gomma respectively. Eight Gandas were randomly selected from Kersa and Mana (four each) and five Gandas from Goma district. First, based on probability proportional to size, the sample size was allocated to each district and within district; the sample size was proportionally allocated to each Ganda based on household size. Then, systematic sampling method was employed to select households for an interview. Household heads were considered for interview. However, the spouse was interviewed if household head was not present at the time of visit. Given that urban population approximately accounts for $15 \%$ in Ethiopia [17], to have fair representation, about $15 \%$ of the households were selected from urban settings. For the purpose of this study, urban was defined as an administrative town with municipality service. A total of six (i.e. two in each district) Focus Group Discussions (FGDs) with women and men in separate groups (three in each) were conducted. In addition, 11 key informant interviews, eight in Mana and Goma (four in each) and three interviews in Kersa, were also conducted with various categories of health workers, school teachers and religious leaders, to complement the quantitative data.

\section{Indicators of LLIN coverage and access}

Standard questionnaire adapted from literatures was used to collect data onLLINaccess, ownership, and coverage $[17,18]$. Household and population based indicators were used for assessment of universal coverage of $\operatorname{LLIN}[8,10,11]$.

\section{Household based indicators \\ Proportion of households in the study with at least one LLIN $\left(P_{1}\right)$}

The numerator consists of all households that own at least one insecticide mosquito net and the denominator is the total of number of sampled households.

\section{Proportion of households with at least one LLIN for every two people $\left(\mathrm{P}_{2}\right)$}

The numerator contains all households where the ratio between numbers of LLIN owned, the number of household members is 0.5 or higher and the denominator is the total number of sampled households. This is the indicator of proportion of households having sufficient access to LLIN.

\section{Population-based indicators}

\section{Proportion of population with access to LLIN within the} household $\left(\mathrm{P}_{3}\right)$

The numerator includes all household members in the sample who had access to LLIN assuming each LLIN was used by two people and the denominator is the population in the sample. The calculation of the numerator was performed based on Roll Back Malaria Monitoring and Evaluation Reference Group's (MERG) procedures [11]: first, potential LLIN users were obtained by multiplying the number of LLIN in each household by the factor of two. Second, the access indicator was calculated by dividing the potential LLIN users by the number of members of each household and computing the overall sample mean of that fraction.

\section{Proportion of population sleeping under an LLIN the previous night $\left(\mathrm{P}_{4}\right)$}

The numerator contains all members of the household identified as one of the users of an LLIN based on the listings of member and the denominator is the population included in the sample.

Additionally, two indicators were computed according to MERG's recommendation [11] in order to facilitate interpretation of ownership and use gaps. They include Proportion of households with at least one LLIN for every two people among households owning any LLIN $\left(P_{5}\right)$. This measures the saturation with LLIN for households with any LLIN. And, the inverse of $\mathrm{P}_{5}\left(1-\mathrm{P}_{5}\right)$ gives the intra-household ownership gap.

Proportion of population sleeping under an LLIN the previous night among those with access $\left(\mathrm{P}_{6}\right)$. The fact that method of calculating the access indicator does not allow allocation of access to specific individuals within the household, $\mathrm{P}_{6}$ was calculated for the overall population included in the study by dividing the number of people who slept under LLIN the previous night to the total population who had access. The inverse of this indicator $\left(1-\mathrm{P}_{6}\right)$ gives the use gap.

\section{Data collection}

The quantitative data were collected by experienced and trained field enumerators. The tool was translated into Afan Oromo (local language) and pretested on $5 \%$ of the sample size in similar setting. The whole process of data collection was closely supervised by three academic staffs of Jimma University. The FGDs and the key informant interviews were conducted by experienced public health professionals who had master's degree of qualifications. 


\section{Data analysis}

The data were analyzed using SPSS version 17.0 software package. Proportions were computed for each indicator and presented in tables and figures. Binary logistic regression was applied to determine the association of background factors with LLIN use. A $95 \%$ confidence interval and level of significance less than 0.05 were used during the analysis. The data from FGDs and the interviews were transcribed verbatim and then translated into English, and finally triangulated with quantitative findings.

\section{Ethical consideration}

The study was approved by the research ethics committee of Jimma University (Ref: RPGC/260/2013). The purpose of the study was explained to each respondent and informed verbal consent was obtained from all respondents.

\section{Results}

Demographic characteristics of the respondents A total of 798 households participated in the study giving a response rate of $95.7 \%$ (Table 1). A total of 4107

Table 1 Demographic characteristic of the respondents, Jimma, Jan 2014

\begin{tabular}{|c|c|c|c|}
\hline \multicolumn{2}{|c|}{ Demographic characteristics } & \multirow{2}{*}{$\begin{array}{l}\text { Frequency } \\
256\end{array}$} & \multirow{2}{*}{$\frac{\text { Percent }}{32.1}$} \\
\hline Districts & Kersa & & \\
\hline & Mana & 218 & 27.4 \\
\hline & Goma & 324 & 40.5 \\
\hline \multirow[t]{2}{*}{ Residence } & Urban & 131 & 16.4 \\
\hline & Rural & 667 & 83.6 \\
\hline \multirow[t]{2}{*}{ Sex of respondents } & Male & 401 & 50.3 \\
\hline & Female & 397 & 49.7 \\
\hline \multirow[t]{3}{*}{ Marital status } & Married & 669 & 83.9 \\
\hline & Widowed & 95 & 11.9 \\
\hline & Others $^{a}$ & 34 & 4.2 \\
\hline \multirow[t]{3}{*}{ Educational status } & Cannot read and write & 399 & 50.0 \\
\hline & Read and write & 214 & 26.8 \\
\hline & Formal education & 185 & 23.2 \\
\hline \multirow[t]{3}{*}{ Religion } & Muslim & 640 & 80.2 \\
\hline & Orthodox & 144 & 18.0 \\
\hline & Protestant & 14 & 1.8 \\
\hline \multirow[t]{4}{*}{ Ethnicity } & Oromo & 650 & 81.4 \\
\hline & Amhara & 58 & 7.3 \\
\hline & Dawuro & 49 & 6.1 \\
\hline & Others $^{b}$ & 41 & 5.2 \\
\hline \multirow[t]{4}{*}{ Occupation } & Farmer & 614 & 77.0 \\
\hline & Private job & 84 & 10.5 \\
\hline & Government employ & 51 & 6.4 \\
\hline & Merchant & 49 & 6.1 \\
\hline
\end{tabular}

${ }^{\mathrm{a}}$ single, divorced, engaged ${ }^{\mathrm{b}}$ keffa, Silte, Yam, Gurage persons were living in these households with average family size of $5.1 \pm 2.1$.

\section{LLIN ownership}

A total of 1174 functional LLINs were accessed in 798 households with mean $1.5 \pm 1.3$ LLINs per household. Less than half $(44.9 \%)$ of the nets were distributed to households very recently (i.e. during the last six months). Overall, $70.9 \%$ (95 \% CI: $67.8-74.1 \%$ ) of the study households had at least one LLIN at the time of the survey (Table 2). However, of the households with at least one LLIN, the coverage of at least one LLIN for every two people was $88.9 \%$ (95 \% CI: 86.3-91.4\%).

\section{Access to and use of LLIN}

At the time of interview, only $491(41.9 \%)$ of the LLINs were hung over the sleeping areas while more than half 641 (54.6\%) of the nets were kept folded. Table 3 shows LLIN access and use by district and place of residence. Nearly half, 51.9 \% (95 \% CI: 50.5-53.5 \%), of the study population (2130/4107) had access to LLIN within the households, with mean access 54.7 \% (95 \% CI: 51.9$57.5 \%)$. LLIN access was the highest in Kersa district and the lowest in Goma. Likewise, the rate of access to LLIN was higher in rural than urban settings.

Regarding LLIN use, 38.4 \% (95 \% CI:36.9-39.9\%) of the study participants slept under LLIN the previous night (Table 3). This net use coverage was higher in rural settings. Of those who had access to the LLIN, the proportion of people who used LLIN the previous night increased to $73.1 \%$ (95 \% CI:71.2-74.9\%) with little variations by district and place of residence. This gave an overall use to access ratio of $70.2 \%$. Despite low access to LLIN in Goma district, the prevalence of LLIN use was relatively higher among those who had access to it (Fig. 2).

\section{LLIN ownership and use gaps}

This study identified two broad LLIN gaps (i.e. ownership and use gaps). Accordingly, $29.1 \%$ (95 \% CI:25.9-32.2\%) of the study households did not have LLIN at the time of the survey. Similarly, intra-household net gap was $11.1 \%$ (95\% CI:8.5-13.6\%), meaning $11.1 \%$ of the households with LLIN did not have sufficient access. Of those households who had any LLIN, $88.9 \%$ (503/566) had sufficient LLIN for usual household members. However, $20.5 \%$ ( $n=103$ ) of these households had excess LLINs (i.e. more than one LLIN for every two people). On the other hand, of those population with sufficient access to LLIN, $26.9 \%$ (95 \% CI:25.0-28.7\%) did not actually use it. Table 4 shows reasons for not using LLIN.

\section{Association of demographic factors with LLIN use}

Table 5 shows results regarding the association of background factors with LLIN use among household members 
Table 2 LLIN ownership by selected background characteristics, Jimma, Jan 2014

\begin{tabular}{|c|c|c|c|c|c|}
\hline \multicolumn{2}{|c|}{ Background characteristics } & \multirow{2}{*}{$\begin{array}{l}\text { HHs at least with } 1 \\
\text { LLIN }\left(\mathrm{P}_{1}\right) \%(95 \% \mathrm{Cl}) \\
96.9(94.7-99.0)\end{array}$} & \multirow{2}{*}{$\begin{array}{l}\text { HHs with at least } 1 \text { LLIN for } \\
\text { every } 2 \text { people }\left(\mathrm{P}_{2}\right) \%(95 \% \mathrm{Cl}) \\
94.5 \text { (91.7-97.2) }\end{array}$} & \multirow{2}{*}{$\begin{array}{l}\text { HHs with at least } 1 \text { LLIN for every } \\
\left.2 \text { people if any LLIN \% (P } P_{5}\right)(95 \% \text { CI)) } \\
97.6(95.7-99.5)\end{array}$} & \multirow{2}{*}{$\begin{array}{l}\text { Number } \\
256\end{array}$} \\
\hline District & Kersa & & & & \\
\hline & Mana & $79.8(70.0-80.0)$ & $66.5(60.2-72.7)$ & $83.3(77.7-88.8)$ & 218 \\
\hline & Goma & $44.6(39.2-50.0)$ & $35.8(30.5-41.0)$ & $80.6(74.1-87.0)$ & 324 \\
\hline \multirow[t]{2}{*}{ Residence } & Urban & $61.1(52.7-69.5)$ & $53.4(44.8-61.9)$ & $87.5(80.2-94.7)$ & 131 \\
\hline & Rural & $72.9(69.5-76.3)$ & $64.9(61.2-68.5)$ & 89.1 (86.3-91.8) & 667 \\
\hline \multirow[t]{2}{*}{ Sex } & Male & $69.0(64.5-73.6)$ & $64.3(59.5-69.0)$ & $88.4(84.7-92.0)$ & 397 \\
\hline & Female & $72.8(68.4-77.2)$ & $61.7(56.9-66.4)$ & $89.4(85.7-93.04)$ & 401 \\
\hline \multirow[t]{2}{*}{ Education } & Yes & $71.0(67.4-74.5)$ & $62.8(58.9-66.6)$ & $88.5(85.5-91.5)$ & 613 \\
\hline & No & $70.8(64.2-77.4)$ & $63.8(56.8-70.7)$ & $90.1(84.9-95.2)$ & 185 \\
\hline Overall & & $70.9(67.8-74.1)$ & $63.0(59.6-66.3)$ & $88.9(86.3-91.4)$ & 798 \\
\hline
\end{tabular}

HHs Households

with full and insufficient access to LLIN. Of household members with full access to LLIN, the use was significantly lower in Mana district (AOR $=0.56$; 95 \% CI: 0.46$0.68, P=0.001)$. Females were 1.52 times more likely to use LLIN compared to males (AOR $=1.52$; 95 \% CI:1.251.83, $P=0.001)$. On the other hand, children aged $0-4$ years $(\mathrm{AOR}=2.28 ; 95 \% \mathrm{CI}: 1.47-3.53, P=0.001)$ and $5-9$ years $(\mathrm{AOR}=1.70 ; 95 \% \mathrm{CI}: 1.11-2.61, p=0.014)$ were more likely to use LLIN compared to older people (age $\geq 50$ years) and other household members. Meanwhile, of the households with insufficient access to LLIN, the use was significantly lower among urban residents (AOR $=0.37$; $95 \%$ CI: 0.18-0.76, $p=0.007$ ). In these households, children aged $0-4$ years were more likely to use LLIN as compared to older people ( $\mathrm{AOR}=7.22 ; 95 \%$ CI:1.74-29.88, $p=0.006$ ). Nevertheless, the LLIN use was significantly lower for younger son/daughter and other family members as compared to heads of households (Table 5).

\section{Discussion}

This study measured LLIN ownership, access and use gaps in malaria endemic settings in Southwestern Ethiopia, based on MERG indicators [10, 11]. It reported LLIN ownership prevalence of $70.9 \%$ which is a better coverage compared to previous national and regional reports [17]. Nevertheless, the result was far behind the national target (100\% coverage) in malarious areas of Ethiopia by 2015 [5]. Even though this finding is nearly similar with some previous reports [19-21], there were many local studies that reported higher LLIN ownership coverage $[15,22,23]$. In one study, $77 \%$ of the households had at least one LLIN [15]; another study reported $85.5 \%$ [22]; and greater than $90 \%$ ownership coverage was also reported elsewhere [23, 24]. In fact, two of the study districts in this study (Kersa and Mana) had quite higher LLIN ownership coverage which could be due to recent distribution of LLIN. On the other hand, LLIN ownership coverage was relatively lower in urban settings (61.1 \% versus72.9 \%) which could be due to limited and inappropriate LLIN distribution campaign in urban areas. This phenomenon could also be related to population dynamics in urban settings. Evidence has also shown that in many African countries LLIN distribution campaigns usually focus on rural settings [25] which might lead to lower LLIN coverage in urban areas. Thus, given the global recommendations to achieve universal LLIN coverage [6], particularly in malaria endemic settings, it is crucial to promote LLIN distribution strategies that fit into to urban contexts.

The ownership coverage indicator is basically relevant to measure the geographical distribution of the nets though it is with limited information about intra-

Table 3 Access and LLIN use by district and place of residence, Jimma, Jan 2014

\begin{tabular}{|c|c|c|c|c|c|c|c|}
\hline \multicolumn{2}{|c|}{ Background characteristics } & \multirow{2}{*}{$\begin{array}{l}\text { Proportion of people } \\
\text { with access to LLIN } \\
\left(P_{3}\right) \%(95 \% \mathrm{Cl}) \\
79.0(77.1-81.3)\end{array}$} & \multirow{2}{*}{$\begin{array}{l}\text { Mean \% of population } \\
\text { with access to an } \\
\text { LLIN \% (95\% CI) } \\
83.5(80.6-86.4)\end{array}$} & \multirow{2}{*}{$\begin{array}{l}\text { LLIN use the } \\
\text { previous Night }\left(\mathrm{P}_{4}\right) \% \\
(95 \% \mathrm{Cl}) \\
57.8(55.3-60.3)\end{array}$} & \multirow{2}{*}{$\begin{array}{l}\text { LLIN use the previous } \\
\text { night if Access }\left(\mathrm{P}_{6}\right) \% \\
(95 \% \mathrm{Cl}) \\
73.1(70.5-75.6)\end{array}$} & \multirow{2}{*}{$\begin{array}{l}\text { Ratio of LLIN use } \\
\text { to access (\%) } \\
69.2\end{array}$} & \multirow{2}{*}{$\begin{array}{l}\text { Number } \\
1450\end{array}$} \\
\hline District & Kersa & & & & & & \\
\hline & Mana & $51.4(48.7-54.6)$ & $57.2(52.4-62.0)$ & $36.0(33.2-38.8)$ & $70.1(66.3-73.8)$ & 62.9 & 1108 \\
\hline & Goma & $26.8(24.7-29.1)$ & $30.4(26.2-34.5)$ & $21.9(19.8-23.9)$ & $81.9(78.2-85.6)$ & 72.0 & 1549 \\
\hline \multirow[t]{2}{*}{ Residence } & Urban & $43.5(39.7-47.3)$ & $46.6(39.5-53.6)$ & $30.4(26.9-33.9)$ & $70.0(64.7-75.27)$ & 65.2 & 667 \\
\hline & Rural & $53.5(52.0-55.4)$ & $56.4(53.3-59.4)$ & $39.9(38.3-41.5)$ & $74.7(72.7-76.6)$ & 70.7 & 3440 \\
\hline Overall & & $51.9(50.5-53.5)$ & $54.7(51.9-57.5)$ & $38.4(36.9-39.9)$ & $73.1(71.2-74.9)$ & 70.2 & 4107 \\
\hline
\end{tabular}




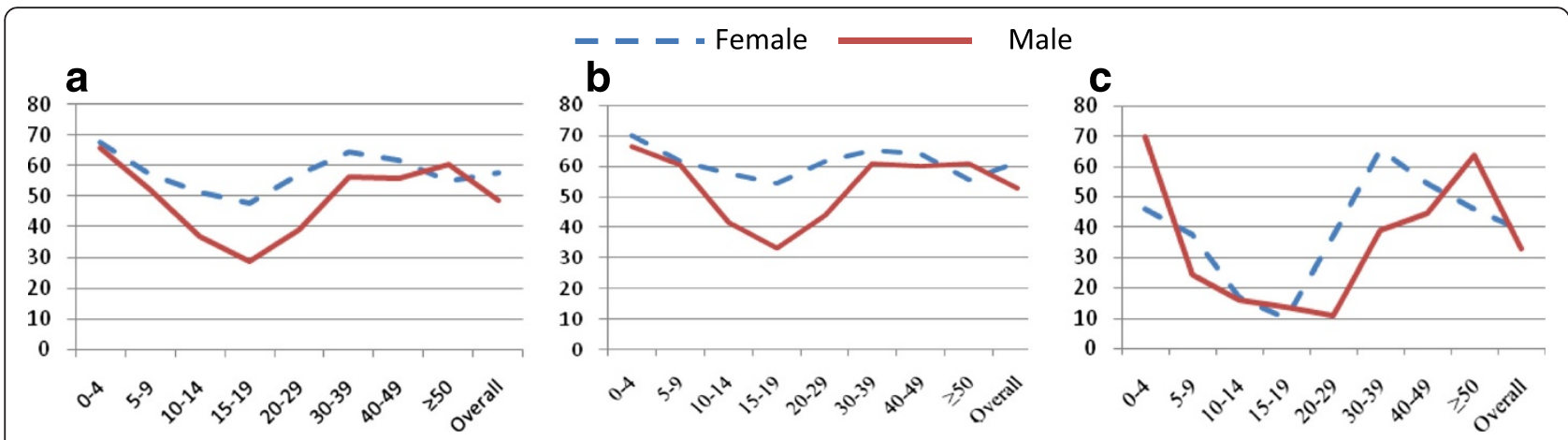

Fig. 2 LLIN use among household members by age and sex, Jimma, Jan 2014. a shows LLIN use in households with at least one LLIN; b shows LLIN use in households with sufficient access and $\mathbf{c}$ indicates LLIN use in households with insufficient LLIN. Overall, except after the age of 48 years, the proportions of females who were using LLIN was higher than that of males in all age groups (a and $\mathbf{b}$ ). Nevertheless, in households with insufficient access to LLIN, males were given priority in under five children implying that insufficient access might have caused gender disparity (c). On the other hand, LLIN use was very low among young people (age 15-19 years) which did not vary by household access to the LLIN. Qualitative data also confirmed that females and children were given priority among household members in general. However, insufficient access to LLIN led to gender disparity

Table 4 Barriers against LLIN use as identified through FGDs and key informant interviews

Characteristics of Nets, and Sleeping Arrangements

- Shape of the net: Given the rectangular nature of the nets, it is not comfortable to hang over sleeping area, 'incompatibility with sleeping arrangements and house style'.

- No bed: Some respondents mentioned people do not use LLIN if they don't have bed or when their bed is under maintenance.

- Sleeping outdoor: Many FGD and key informants mentioned that adolescents often sleep outdoor which often makes LLIN use difficult.

- Perceived low efficacy of LLIN: Most participants argued that unlike past times, the net stopped killing mosquitoes and other insects and as result people throw it away or use for other purpose. "When the chemical in the LLIN is unable to kill mosquito, people may use it for other purposes, as curtain or as cover of other materials." [FGD participant]

- Fear of chemical: "The chemicals in it [LLIN] sometimes cause irritation, cough and itching." [FGD participant]

Seasonality of Mosquitoes Bite

- No mosquito bite: Given the study was conducted during dry seasons, people argued that mosquito does not exist during dry season and there is no need to sleep under the net.

- Saving LLIN for another time: People mentioned that they saved the nets for more risky time. This may confirm the fact that more than half of the nets were kept folded at the time of visit for interview.

Accessibility factors

- Insufficient access to LLIN within households.

- Mal-distribution of LLIN that leads to either scarcity or excess nets

Personal factors

- Lack of awareness and carelessness among families

- Using the nets for other unintended purposes, as 'mattress', 'to cover toilet'

- Low perceived risk of malaria infections

- Throwing away once it is 'thorn out 'and/or 'becomes dirty' household LLIN saturation which provides crucial information to roll out behavioral driven gaps [7]. WHO recommends universal coverage assuming one net for every two people and this goal remains the intended outcome for all areas at risk of malaria $[6,26]$.

Considering one LLIN for every two people, only $63.0 \%$ of the sampled households in this study had enough LLIN for every member of the household. However, analysis of those households who owned any LLIN showed that ownership of at least one LLIN for every two people was increased to $88.9 \%$ with intrahousehold net gap of $11.1 \%$. In contrast, a significant numbers of extra LLINs were noted in large portion of households (20.5\%) suggesting inequitable distribution of LLINs in the community. Households might have saved some of the nets for times of high transmission season or due to fear of shortage of nets which could be accounted for the availability of extra LLIN in some households. In addition, some households might have purchased more nets or there could be a possibility of routine distribution of LLIN through antenatal and immunization programs. This phenomenon was also reported in the qualitative part of this study. Some earlier studies also reported availability of extra LLINs in some households while other families were without any LLIN $[22,27]$.

Another important population based LLIN indicator is the proportion of population with access to LLIN within the household assuming each LLIN was used by two people. Consequently, despite fairly high coverage of ownership of at least one LLIN, nearly half of the population had no access to LLINs. This shows that there a huge access gap in these malaria endemic settings contrary to the WHO universal access coverage and national target $[5,6,10]$. 
Table 5 Association of demographic characteristics with LLIN use the previous night, Jimma, Jan 2014

\begin{tabular}{|c|c|c|c|c|c|c|c|}
\hline \multirow[t]{2}{*}{ Background characteristics } & \multicolumn{4}{|c|}{$\begin{array}{l}\text { HHs with sufficient access to LLIN } \\
\text { LLIN use the previous night }\end{array}$} & \multicolumn{3}{|c|}{$\begin{array}{l}\text { HHs with insufficient access to LLIN } \\
\text { LLIN use the previous night }\end{array}$} \\
\hline & & Yes n (\%) & No n (\%) & AOR $(95 \% \mathrm{Cl})$ & Yes n (\%) & No n (\%) & AOR $(95 \% \mathrm{Cl})$ \\
\hline \multirow[t]{3}{*}{ District } & Kersa & $821(62.0)$ & $504(38.0)$ & 1 & $17(36.2)$ & $30(63.8)$ & 1 \\
\hline & Mana & $322(47.9)$ & $350(52.1)$ & $0.56(0.46-0.68)^{*}$ & $77(41.2)$ & $110(58.8)$ & $1.36(0.63-2.94)$ \\
\hline & Goma & $285(57.3)$ & $212(42.7)$ & $0.82(0.66-1.01)$ & $55(30.1)$ & $128(69.9)$ & $0.68(0.31-1.50)$ \\
\hline \multirow[t]{2}{*}{ Residence } & Urban & $185(57.5)$ & $137(42.5)$ & $0.99(0.78-1.27)$ & $18(28.1)$ & $46(71.9)$ & $0.37(0.18-0.76)^{*}$ \\
\hline & Rural & $1243(57.2)$ & $929(42.8)$ & 1 & $131(37.1)$ & $222(62.9)$ & 1 \\
\hline \multirow[t]{2}{*}{ Sex } & Female & $783(61.3)$ & $494(38.7)$ & $1.52(1.25-1.83)$ & $75(38.9)$ & $118(61.1)$ & $0.98(0.54-1.76)$ \\
\hline & Male & $645(53.0)$ & $572(47.0)$ & 1 & 74(33.0) & $150(67.0)$ & 1 \\
\hline \multirow[t]{8}{*}{ Age } & $0-4$ & $220(68.3)$ & $102(31.7)$ & $2.28(1.47-3.53)^{*}$ & $27(62.8)$ & $16(37.2)$ & $7.22(1.74-29.88)^{*}$ \\
\hline & $5-9$ & $232(61.1)$ & $148(38.9)$ & $1.70(1.11-2.61)$ & $26(31.7)$ & $56(68.3)$ & $1.69(0.43-6.53)$ \\
\hline & $10-14$ & $195(49.4)$ & $200(50.6)$ & $1.02(0.67-1.56)$ & $11(16.4)$ & $56(83.6)$ & $0.68(0.16-2.82)$ \\
\hline & $15-19$ & $118(44.0)$ & $150(56.0)$ & $0.83(0.53-1.28)$ & $6(12.0)$ & $44(88.0)$ & $0.45(0.09-2.19)$ \\
\hline & $20-29$ & $212(54.1)$ & $180(45.9)$ & $1.02(0.72-1.46)$ & $13(23.6)$ & $42(76.4)$ & $0.52(0.15-1.83)$ \\
\hline & $30-39$ & $170(63.2)$ & $99(36.8)$ & $1.20(0.84-1.71)$ & $30(56.6)$ & $23(43.4)$ & $0.69(0.25-1.87)$ \\
\hline & $40-49$ & $117(62.2)$ & $71(37.8)$ & $1.17(0.79-1.73)$ & $14(48.3)$ & $15(51.7)$ & $0.42(0.14-1.24)$ \\
\hline & $\geq 50$ & $164(58.6)$ & $116(41.4)$ & 1 & $22(57.9)$ & $16(42.1)$ & 1 \\
\hline \multirow[t]{4}{*}{ Relationship to household head } & Head & $276(62.3)$ & $167(37.7)$ & 1 & $32(54.2)$ & $27(45.8)$ & 1 \\
\hline & Spouse & $278(63.3)$ & $161(36.7)$ & $0.74(0.53-1.04)$ & $39(66.1)$ & $20(33.9)$ & $1.87(0.68-5.18)$ \\
\hline & Son/daughter & $794(55.9)$ & $627(44.1)$ & $0.54(0.38-0.77)^{*}$ & $74(27.2)$ & $198(72.8)$ & $0.14(0.04-0.48)^{*}$ \\
\hline & Others & $80(41.9)$ & $111(58.1)$ & $0.32(0.21-0.50)^{*}$ & $4(14.8)$ & $23(85.2)$ & $0.08(0.02-0.35)$ \\
\hline
\end{tabular}

*Significant at $p<0.05$

In line with global malaria control and elimination efforts, Ethiopia has planned for malaria elimination which demands achieving and sustaining universal coverage of at least $80 \%$ of all malaria interventions coverage including LLIN usage. This is necessary to move away from scaling-up for impact (SUFI) to sustained control and pre-elimination phase [10]. However, based on the findings, the progress towards the national target could be very slow as the current coverage is far from the target set to be achieved by 2015 [10]. In order to achieve sustainable coverage of LLIN, WHO recommends that countries should apply a combination of mass free distributions and continuous distributions through multiple channels, particularly through antenatal and immunization programs [28].

Use of LLINs is one of the most cost-effective interventions, and high use rates are a central goal in malaria control programs in malaria endemic settings [7, 8, 10, 28]. However, in this study, only $38.4 \%$ of the household members slept under LLIN the night before the survey. The utilization rate was critically low in Goma district where only $21.9 \%$ of the people reported sleeping under LLIN the previous night. The lack of access to LLIN could account for the low utilization rate in this district. The fact that more than half of the LLINs were kept unused at the time of the visit could also justify the low LLIN use coverage in this community which is also documented in earlier study [15]. Low risk perceptions, lack of access, negligence, saving and misusing nets, technical difficulties related to hanging, and perceived degraded efficacy of the nets were also cited reasons for poor utilization of LLIN. Nevertheless, consistent with previous reports from Ethiopia [17, 18], sleeping under LLIN was relatively higher among pregnant women and under five children. In contrast, it is lower compared to the results from some earlier studies [15-24, 27, 29-32]. Lower LLIN usage in the current study could be attributed to the time of the study (dry season) in which malaria transmission is low and people might not use LLIN. This phenomenon was also noted in the qualitative investigations in which several participants mentioned that people were less concerned about malaria during dry season when people are less likely to use mosquito nets. In unstable malaria transmission contexts, such as in the current study, this finding has important implications for malaria program in that it signifies the need to promote continued usage of LLIN. On the other hand, the deterioration of LLIN in both quality of insecticide and physical integrity might also be negatively impacted on LLIN use and its effectiveness. Even though evidence from the qualitative part of the study provided some insight about the converse relationship between 
perceived quality of LLIN and its use, this study did not provide in-depth information on this issue, and more studies could be important to gain further understanding about it.

Excluding households who did not own any LLIN from analysis, previous night LLIN use increased to $52.9 \%$ among the study households with at least one LLIN and to 73. One percent among households with sufficient access with overall ratio of use to access being $70.2 \%$. This ratio allowed identifying the difference between non-use due to lack of access and non-use due to behavioral failure. Hence, a significant portion of the population who had access to LLIN did not actually use it, showing a substantial gap between access and actual use. Behavioral failure was quite high considering the general expectation that access led to higher user coverage [33]. Nevertheless, despite low LLIN access rate in Goma district, there was a strong habit of sleeping under LLIN when there was access to LLIN. This entails non-use was mostly linked to lack of nets than behavioral failure in this district. This fact may indicate existence of good awareness about malaria and LLIN use in this district. With regard to gender, females were more likely to use LLIN than males across all age groups in households with sufficient access to LLIN. Moreover, under five children were also more likely to use LLIN than younger adults and older people. This could reflect the success of the conventional communication approach that encourages LLIN use by women and children. Of course, it would be a logical argument as women often sleep under the same net with children.

The rate of net use was consistently lower among people in the age group of 10-19 years whether the households had sufficient access or not, which is also documented in some previous studies [32, 34, 35]. The fact that LLIN use in this age group did not depend on LLIN access reflects existence of huge behavioral failure in this specific age group calls for targeted behavioral change communication interventions. Results from the qualitative component of the study also complemented this finding in that older children (usually aged 10-20 years) often sleep outdoor which makes LLIN use less likely. Some recent reports revealed that the indoor biting behaviors of mosquitoes have been shifting to outdoor, and outdoor sleeping significantly increases the risk of malaria infection [36, 37]. The outdoor sleeping practice could be a potential challenge to universal LLIN usage which in turn increases residual transmission to be maintained in the community despite high indoor LLIN utilization. This requires reorientation of the conventional LLIN promotion approaches which focused mostly on indoor interventions with particular attention to women and children.
In households with insufficient access to LLIN, children were more likely to sleep under LLIN although males were given priority for LLIN use. This implies that insufficient access to the LLIN might have led to gender difference in LLIN use which was also cited in another study [38]. Perhaps, gender-specific behavior change communication intervention strategies could help to promote gender equity. It is also important to look at specific population segments and groups when designing and implementing a behavior change intervention strategy for LLIN promotion.

Evidence has shown that BCC plays a vital role in creating demand and increasing the LLIN use; makes families use their nets regularly and care and repair them [39]. Furthermore, BCC continues to play a key role even when the risk of malaria infections is greatly reduced. BCC can serve as a tool in malaria elimination efforts by targeting hot-spot reservoirs of infection which often overlooked during planning in control programs $[39,40]$. Certainly, the prospect of achieving and sustaining universal user coverage of LLIN in malaria endemic areas heavily depends on a solid investment and welldesigned $\mathrm{BCC}$ interventions.

\section{Limitations of the study}

Since the study was conducted during low malaria transmission season, the finding may not reflect year round situations of LLIN use among the populations. Furthermore, this study was limited to a small geographical area with similar seasonal and unstable malaria transmission and it may not reflect LLIN use in other eco-epidemiologic settings in Ethiopia.

\section{Conclusions}

Ownership coverage of LLINs was lagging behind the national and global targets in the study community, characterized by lower access rate with a relatively wide intra-household net coverage gap. There was a wide gap between ownership of nets and actual use. However, higher access rate was basically associated with increased net use although not to the expected level. It was noted that low access to LLIN leads to gender disparity particularly among children. On the other hand, behaviordriven non-use of LLIN and non-use due to lack of the nets remained significant constraints to universal usage of nets in the study setting. Given that LLIN access to and use coverage was far short of the targets, it conveys two important messages to the malaria control program for speedy move towards elimination and eventual eradication: 1) achieving and maintaining universal LLIN coverage is crucial, 2) addressing behavior-driven nonuse through well designed and tailored behavioral change interventions. Further, it calls for the need to go beyond the traditional messaging approach to evidence based practice by taking into account gender and age 
specific tailored interventions. Finally, it should be noted that this study provided cross-sectional evidence on LLIN access and use which is useful information to inform LLIN distribution and usage programming. It also indicates the need to conduct large scale study to model the variations in LLIN access and usage gaps.

\section{Abbreviations}

BCC: behavior change communication; FGDs: focus group discussions; HEWs: health extension workers; IRS: indoor residual spray; LLINs: long-lasting insecticidal nets; MERG: malaria evaluation reference group; PMI: president's malaria initiative; RBM: roll back malaria; WHO: World Health Organization.

\section{Competing interests}

The authors declared that they have no competing interests.

\section{Authors' contributions}

$\mathrm{ZB}, \mathrm{MS}$, and LA conceived the study; was involved in the design and conduct of the study. ZB write the report and MS, LA, GA, GD, DY and YY reviewed the report. ZB drafted the manuscript and MS, LA, GD, GA, DY, and YY critically reviewed the manuscript for intellectual content. ZB revised the manuscript and all authors read and approved the final version of the manuscript.

\section{Acknowledgements}

This study received financial support from the United States Agency for International Development (USAID)-Ethiopia. The authors appreciate the study participants for their cooperation in providing the necessary information. We acknowledge Jimma University, local administrators, and the community for their strong support during the survey. We would like to extend our sincere thanks to Dr kefelegn Getahun who helped us in developing map of the study area.

\section{Disclaimer statements}

USAID staff must include, except in circumstances described below with regard to employment status at the time of research and publication, the following disclaimer in publications they author: "The views and opinions expressed in this paper are those of the authors and not necessarily the views and opinions of the United States Agency for International Development."

\section{Author details}

'Department of Health Education and Behavioral Sciences, Jimma University, Jimma, Ethiopia. ${ }^{2}$ President's Malaria Initiative, United States Agency for International Development, Addis Ababa, Ethiopia. ${ }^{3}$ Abt Associates African Indoor Residual Project, Addis Ababa, Ethiopia. ${ }^{4}$ Department of Medical Laboratory Sciences, Jimma University, Jimma, Ethiopia.

\section{Received: 5 June 2015 Accepted: 22 December 2015}

\section{Published online: 29 December 2015}

\section{References}

1. WHO: World Malaria Report 2014 [Internet]. WHO. [Cited 2015 Mar 20]. Available from: http://www.who.int/malaria/publications/world_malaria_ report_2014/report/en/

2. PMI: President's Malaria Initiative Strategy 2015-2020, 2015 [internet]. PMI. [cited 2015 March 20]. Available from:http://www.malariaeradication.org/ knowledge-hub/presidents-malaria-initiative-malaria-strategy-2015-2020

3. Federal Ministry of Health of Ethiopia. Health and health related indicators. Addis Ababa: Ministry of Health [Ethiopia]; 2013.

4. Federal Ministry of Health of Ethiopia. Health sector development program IV. Addis Ababa: Ministry of Health [Ethiopia]; 2010

5. Federal Ministry of Health of Ethiopia. National strategic plan for malaria prevention control and elimination in Ethiopia 2011 - 2015. Addis Ababa: Ministry of Health [Ethiopia]; 2010.

6. WHO: WHO recommendations for achieving universal coverage with long lasting insecticidal nets in malaria control. Geneva, World Health Organization, 2013[internet]. WHO. [cited 2015 January 22]. Available form: http://www.who.int/malaria/publications/atoz/who_recommendation_ coverage_llin/en/
7. WHO. Insecticide-Treated Mosquito Nets: A WHO Position Statement. Geneva: World Health Organization; 2007.

8. Roll Back Malaria: Guidelines for core population-based indicators. Geneva: RBM Technical Working Paper No. 1; 2009 [internet]. RBM.[cited 2015 March 10]. Available from: http://reliefweb.int/sites/reliefweb.int/files/resources/ AC719D00E5DE6F5D492575B3001BE5A3-RMB-guideline-20009.pdf.

9. Hetzel MW, Gideon G, Lote N, Makita L, Siba PM, Mueller I. Ownership and usage of mosquito nets after four years of large-scale free distribution in Papua New Guinea. Malar J. 2012;11:192.

10. Roll Back Malaria Partnership: Changes to guidance for vector control indicators. New York, USA: Meeting report of the 17th MERG meeting, 15-17.6; 2011 [internet]. RBM. [cited 2015 March 21]. Available from: http://www.rollbackmalaria.org/files/files/partnership/wg/wg_monitoring/ docs/17merg_meeting_report.pdf.

11. Measure DHS: Malaria indicator survey: tabulations for key malaria indicators. Calverton, Maryland, USA; 2012 [internet]. [Cited 2015 March 20]. Available from: http://malariasurveys.org/toolkitfiles/ 10\%20Tabulations\%20for\%20Key\%20Malaria\%20Indicators.pdf.

12. Federal Democratic Republic of Ethiopia. Summary and statistical report of the population and housing census. Addis Ababa: Population Census Commission; 2008.

13. Checchi F, Cox J, Balkan S, Tamrat A, Priotto G, Alberti KP, et al. Malaria Epidemics and Interventions, Kenya, Burundi, Southern Sudan, and Ethiopia, 1999-2004. Emerg Infect Dis. 2006;12(10):1477-85.

14. Ministry of Health [Ethiopia]. Malaria [Internet]. [cited 2015 Nov 5]. Available from: http://www.moh.gov.et/malaria.

15. Deribew A, Alemseged F, Birhanu Z, Sena L, Tegegn A, Zeynudin A, et al. Effect of training on the use of long-lasting insecticide-treated bed nets on the burden of malaria among vulnerable groups, south-west Ethiopia: baseline results of a cluster randomized trial. Malar J. 2010:9(1):121.

16. Karunamoorthi K, Bekele M. Changes in Malaria Indices in an Ethiopian Health Centre: A Five Year Retrospective Analysis. Health Scope. 2012;1(3):118-26.

17. Ethiopian Health and Nutrition Research Institute \& partners. Ethiopia National Malaria Indicator Survey 2011. Addis Ababa: Ethiopian Health and Nutrition Research Institute; 2012

18. Central Statistical Agency of Ethiopia. Ethiopian demographic and health survey: preliminary report. Maryland: ICF Macro Calverton; 2011.

19. Hightower A, Kiptui R, Manya A, Wolkon A, VandenEng J, Hamel M, et al. Bed net ownership in Kenya: the impact of 3.4 million free bed nets. Malar J. 2010;9:183.

20. Kulkarni MA, Eng JV, Desrochers RE, Cotte AH, Goodson JL, Johnston A, et al. Contribution of Integrated Campaign Distribution of Long-Lasting Insecticidal Nets to Coverage of Target Groups and Total Populations in Malaria-Endemic Areas in Madagascar. Am J Trop Med Hyg. 2010;82(3): $420-5$.

21. Terlouw DJ, Morgah K, Wolkon A, Dare A, Dorkenoo A, Eliades MJ, et al. Impact of mass distribution of free long-lasting insecticidal nets on childhood malaria morbidity: the Togo National Integrated Child Health Campaign. Malar J. 2010:9:199.

22. Haileselassie B, Ali A. Assessment of Insecticide Treated Nets Coverage for Malaria Control in Kafta-Humera District, Tigray: Possession versus Use by High-risk Groups. Ethiop J Health Dev. 2008;22(3):259-67.

23. Baume CA, Reithinger $\mathrm{R}$, Woldehanna $\mathrm{S}$. Factors associated with use and non-use of mosquito nets owned in Oromia and Amhara Regional States, Ethiopia. Malar J. 2009:8(1):264

24. Atieli HE, Zhou G, Afrane Y, Lee M-C, Mwanzo I, Githeko AK, et al. Insecticide-treated net (ITN) ownership, usage, and malaria transmission in the highlands of western Kenya. Parasit Vectors. 2011;4(1):113.

25. Strachan L, Strachan C. Stakeholder review of experiences in urban Long Lasting Insecticidal Net (LLIN) campaign distributions [internet]. Malaria Consortium Africa. 2012. [cited 2015 Mar 29]. Available from: http://www.vector-works.org/resources/experiences-in-llin-urbandistribution-campaigns-stakeholder-review/.

26. WHO: Malaria report [Internet]. WHO. [cited 2015 Mar 24]. Available from: http://www.who.int/immunization/programmes_systems/interventions/ malaria/en/

27. Zhou G, Li JS, Ototo EN, Atieli HE, Githeko AK, Yan G. Evaluation of universal coverage of insecticide-treated nets in western Kenya: field surveys. Malar J. 2014;13(1):351

28. WHO. Malaria Policy Advisory Committee to the WHO: conclusions and recommendations of March 2013 meeting. Malar J. 2013;12:213. 
29. Deribew A, Birhanu Z, Sena L, Dejene T, Reda AA, Sudhakar M, et al. The effect of household heads training on long-lasting insecticide-treated bed nets utilization: a cluster randomized controlled trial in Ethiopia. Malar J. 2012;11(1):99

30. Githinji S, Herbst S, Kistemann T, Noor AM. Mosquito nets in a rural area of Western Kenya: ownership, use and quality. Malar J. 2010;9:250.

31. Garley AE, Ivanovich E, Eckert E, Negroustoueva S, Ye Y. Gender differences in the use of insecticide-treated nets after a universal free distribution campaign in Kano State, Nigeria: post-campaign survey results. Malar J. 2013;12(1):119.

32. Kilian A, Koenker H, Baba E, Onyefunafoa EO, Selby RA, Lokko K, et al. Universal coverage with insecticide-treated nets - applying the revised indicators for ownership and use to the Nigeria 2010 malaria indicator survey data. Malar J. 2013;12(1):314.

33. Eisele TP, Keating J, Littrell M, Larsen D, Macintyre K. Assessment of Insecticide-Treated Bednet Use among Children and Pregnant Women across 15 Countries Using Standardized National Surveys. Am J Trop Med Hyg. 2009;80(2):209-14.

34. Bennett A, Smith SJ, Yambasu S, Jambai A, Alemu W, Kabano A, et al. Household possession and use of insecticide-treated mosquito nets in Sierra Leone 6 months after a national mass-distribution campaign. PLoS ONE. 2012;7(5):e37927.

35. Graves PM, Ngondi JM, Hwang J, Getachew A, Gebre T, Mosher AW, et al. Factors associated with mosquito net use by individuals in households owning nets in Ethiopia. Malar J. 2011;10(1):354.

36. Russell TL, Govella NJ, Azizi S, Drakeley CJ, Kachur SP, Killeen GF. Increased proportions of outdoor feeding among residual malaria vector populations following increased use of insecticide-treated nets in rural Tanzania. Malar J. 2011;10(1):80.

37. Reddy MR, Overgaard HJ, Abaga S, Reddy VP, Caccone A, Kiszewski AE, et al. Outdoor host seeking behaviour of Anopheles gambiae mosquitoes following initiation of malaria vector control on Bioko Island, Equatorial Guinea. Malar J. 2011;10(1):184.

38. Renne EP, Kirby K, Akkineni R. Bednet Use and Malaria Knowledge in Zaria City, Nigeria. J Int Instit. 2008;15(2):5.

39. Koenker H, Keating J, Alilio M, Acosta A, Lynch M, Nafo-Traore F. Strategic roles for behaviour change communication in a changing malaria landscape. Malar J. 2014;13(1):1.

40. Cotter C, Sturrock HJW, Hsiang MS, Liu J, Phillips AA, Hwang J, et al. The changing epidemiology of malaria elimination: new strategies for new challenges. Lancet. 2013;382(9895):900-11.

\section{Submit your next manuscript to BioMed Central and we will help you at every step:}

- We accept pre-submission inquiries

- Our selector tool helps you to find the most relevant journal

- We provide round the clock customer support

- Convenient online submission

- Thorough peer review

- Inclusion in PubMed and all major indexing services

- Maximum visibility for your research

Submit your manuscript at www.biomedcentral.com/submit

C Biomed Central 